\title{
Neutron beam performance of iBNCT as linac-based neutron source for boron neutron capture therapy
}

\author{
Hiroaki Kumada ${ }^{1, *}$, Susumu Tanaka ${ }^{1}$, Fujio Naito $^{2}$, Toshikazu Kurihara ${ }^{2}$, Takashi Sugimura ${ }^{2}$, Hideyuki Sakurai ${ }^{1}$, \\ Akira Matsumura ${ }^{1}$, and Takeji Sakae ${ }^{1}$
}

${ }^{1}$ Faculty of Medicine, University of Tsukuba, 1-1-1, Tennodai, Tsukuba, Ibaraki, 305-8575, Japan

${ }^{2}$ High Energy Accelerator Research Organization, 1-1, Oho, Tsukuba, Ibaraki, 305-0801, Japan

\begin{abstract}
The linac-based neutron source "iBNCT" developed by the Tsukuba team has been shown to generate a large intensity of neutrons. To confirm the applicability of the device to BNCT, several characteristic measurements have been implemented. In a water phantom experiment, when the accelerator was operated with an average current of $1.4 \mathrm{~mA}$, the maximum thermal neutron flux was approximately $7.8 \times 10^{8}\left(\mathrm{~cm}^{-2} \mathrm{~s}^{-1}\right)$. From estimation of the stability of the linac, the results demonstrate its applicability to actual treatment.
\end{abstract}

\section{Introduction}

In recent years, the Boron Neutron Capture Therapy (BNCT) has attracted attention as a treatment method for intractable cancers and recurrent cancers. BNCT is a binary system, based on the nuclear reaction between lowenergy neutrons and the boron isotope boron-10 accumulated in the tumor cells [1]. In principle, BNCT can destroy cancer cells selectively while preventing serious damage to normal tissues, even if the cancer cells invade them. BNCT requires the availability of highintensity neutrons. Thus far, clinical studies of BNCT have been conducted by using research reactors. And recent treatments have used epithermal neutrons $(0.5 \mathrm{eV}$ $<$ Energy $<10 \mathrm{keV}$ ) to deliver therapeutic doses to deeper regions within the human body. The clinical results using reactors have demonstrated the effectiveness of BNCT for treating intractable cancers [2]. However, the therapy has not been established yet since its implementation requires the use of reactors.

The recent progress in the development of technologies for accelerator-based neutron sources have rendered it possible to produce enough neutrons for BNCT using a compact accelerator, which can be installed in hospitals. Therefore, it is expected that BNCT could be established as a general cancer treatment in the near future. Based on this background, many development projects for the accelerator-based neutron source applicable to BNCT are being undertaken in the world [3]. The iBNCT project, a development project for the linacbased neutron source for BNCT, is currently underway [4]. The accelerator of the iBNCT has adopted a RadioFrequency Quadrupole Linac (RFQ) and a Drift Tube Linac (DTL) type linac. Fig. 1 shows the linac of the iBNCT. The linac accelerates protons to $8 \mathrm{MeV}$ with pulsed mode operation and the average current that can be accelerated has been designed to be $5 \mathrm{~mA}$ or more. When the repetition cycle is $50 \mathrm{~Hz}$, the duty is $5.0 \%$ and the average current is approximately $1.4 \mathrm{~mA}$. For the target material for neutron generation, iBNCT has adopted beryllium. Table 1 gives the main specifications for the iBNCT. Each value in parentheses in the table is a target value in the design stage.

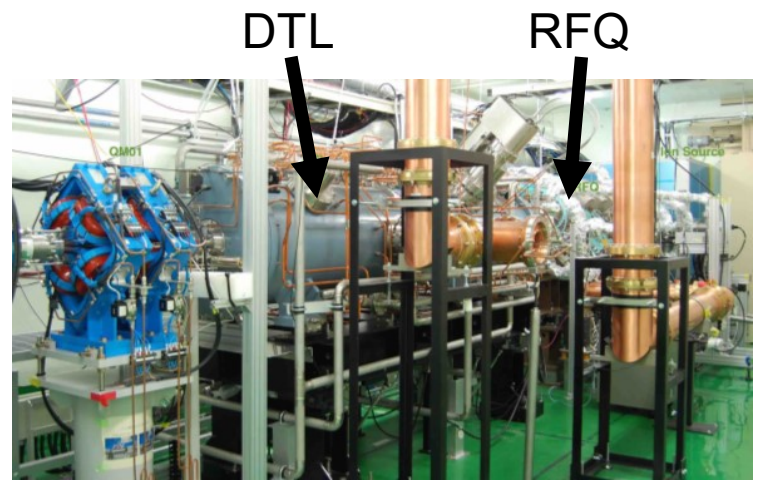

Fig. 1. An RFQ and a DLT-type linac-based neutron source for BNCT at the University of Tsukuba, Japan.

Table 1. Main specifications for the demonstrator of iBNCT.

\begin{tabular}{|c|c|}
\hline Accelerator type & RFQ + DTL proton linac \\
\hline Proton energy & $8 \mathrm{MeV}$ \\
\hline Peak proton current & $>28 \mathrm{~mA}(50 \mathrm{~mA})$ \\
\hline Ave. proton current & $>1.4 \mathrm{~mA}(>5 \mathrm{~mA})$ \\
\hline Pulse width & Max. 1 millisecond \\
\hline Repetition cycle & $50 \mathrm{~Hz}(>125 \mathrm{~Hz})$ \\
\hline Duty & $>5.0 \%(>12.5 \%)$ \\
\hline Target material & Beryllium \\
\hline Accelerator size & $<8 \mathrm{~m}$ \\
\hline
\end{tabular}

The construction of the demonstrator of the iBNCT was completed in 2016. It has been successful for generating

* Corresponding author: kumada@pmrc.tsukuba.ac.jp 
neutrons at that time, albeit having low intensity. Subsequently, various improvements and modifications have been carried out to enhance neutron intensity. At present, in order to conduct clinical trials using the device in the near future, several characteristic measurements of the neutron beam of the iBNCT device are being carried out.

\section{Materials and Methods}

\subsection{Measurement of the distributions thermal neutron flux and gamma-ray dose rate in a water phantom}

It is required to grasp the dose distribution administered around the tumor region when the neutron beam emitted from the iBNCT is irradiated to a human body. For this, several neutron beam irradiation experiments using a water phantom have been performed. In the experiments, a $200 \times 200 \times 200 \mathrm{~mm} 3$ rectangular phantom made of polymethyl methacrylate has been used. The thickness of each wall of the phantom except for the beam side wall is $10 \mathrm{~mm}$, and the thickness of the beam-side wall is $3 \mathrm{~mm}$. In the experiments, several detectors for radiation measurements have been set up inside the phantom that is filled with water. The phantom is positioned to irradiation position in the irradiation room of the iBNCT facility and is irradiated by the neutron beam. In Fig. 2-(a), the phantom is shown and in Fig. 2-(b), the experimental setup is shown with the phantom located at the irradiation position in the irradiation room.

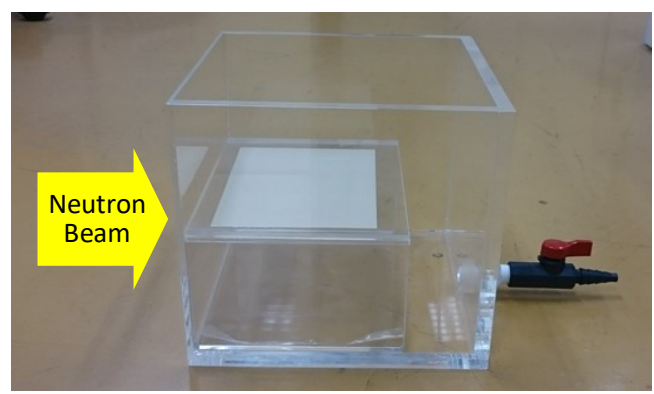

(a) $20 \times 20 \times 20 \mathrm{~cm}^{3}$ rectangular water phantom

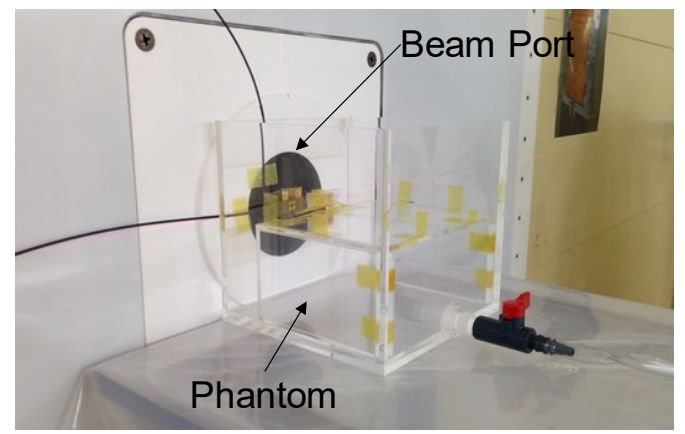

(b) Experimental situation with the phantom

Fig. 2 (a) Cubic water phantom (b) experimental setup with the phantom at the irradiation position

To estimate the dose distribution for BNCT, distributions for thermal neutron flux and gamma-ray dose rate, which are the fundamental dose elements of BNCT, have been measured.

For the measurement of thermal neutron flux, the activation foil method [5], has been adapted in which pure gold (Au-197) is used. A few Au wires of 10-mm length each have been placed on the beam axis inside the phantom. Fig. 3-(a) shows the schematic image of the arrangement of the Au wires in the phantom.

In the experiments, the linac is operated with an average current of $1.4 \mathrm{~mA}$. After irradiation, the radioactivity of each gold wire has been measured using a germanium detector and finally, the distributions of the thermal neutron flux have been determined.

For the measurement of the distribution for the gamma-ray dose rate, a thermoluminescent dosimeter (TLD) has been used as the gamma-ray dose detector [6]. Several TLDs have been placed on the beam axis in the phantom as shown in Fig. 3-(b). After imparting neutron beam irradiation to the phantom, measurements have been taken from each TLD and finally, the distribution for the gamma-ray dose rate has been determined.

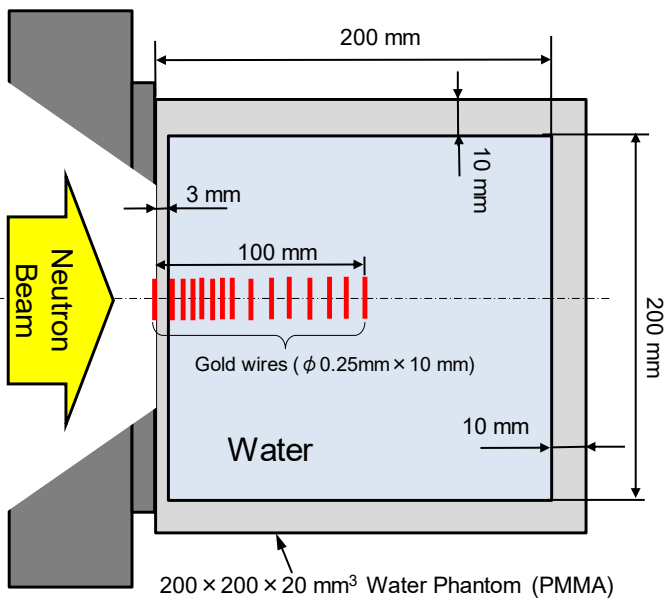

(a) Thermal neutron flux measurement

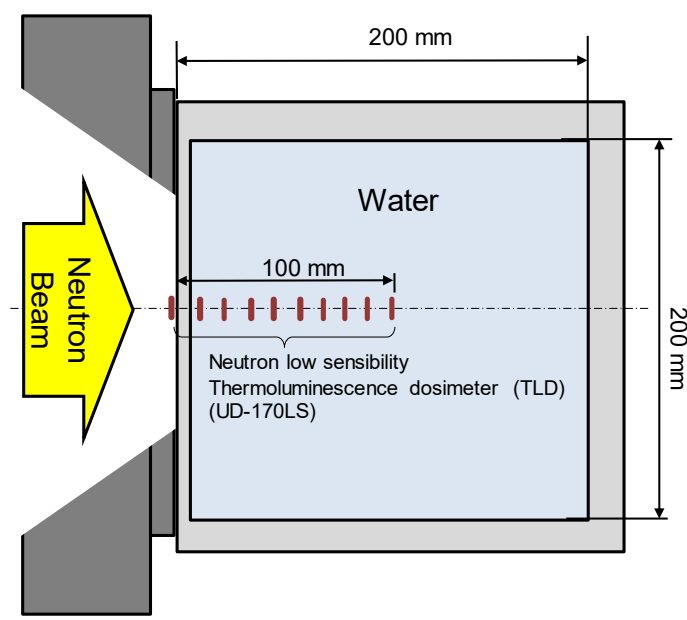

(b) Gamma-ray dose rate measurement

Fig. 3 Experimental conditions for the water phantom irradiation to measure the distributions for (a) thermal neutron flux and (b) gamma-ray dose rate 


\subsection{Dose estimation using Monte Carlo transport code}

To estimate distributions of the equivalent doses for a tumor and for normal tissues in the phantom by neutron irradiation, Monte Carlo solusion has been carried out. For estimation, the Particle and Heavy Ion Transport Code System (PHITS) has been used as a Monte Carlo code. And for the nuclear data library required for the Monte Carlo calculation, JENDL-4.0 has been used. In the calculation, the specifications of the phantom experiment such as the geometry of the iBNCT facility and the phantom and the neutron beam characteristics have been reconstructed properly using PHITS. In the PHITS calculation, two-dimensional (2D) distributions for multiple absorbed doses consisting of boron-10 dose, nitrogen dose, hydrogen dose, and gamma-ray dose have been determined. The four absorbed doses are the basis of the equivalent dose [7]. In addition to the absorbed doses, the distributions for thermal neutron flux and for gammaray dose rate in the phantom have also been calculated in order to determine a normalization factor for the calculation values.

The 2D distributions for equivalent doses (Gy-Eq) in the phantom have been determined by combining with the calculated absorbed doses. Equation (1) is generally used for BNCT dosimetry [7].

$$
\begin{aligned}
& E D(\mathrm{~Gy}-\mathrm{Eq})=\left(C_{B} \cdot D_{B, p p m} \times C B E_{B}\right) \\
& +\left(D_{N} \times R B E_{N}\right)+\left(D_{H} \times R B E_{H}\right)+\left(D_{\gamma}\right)
\end{aligned}
$$

where $\mathrm{ED}$ is an equivalent dose rate. $\mathrm{D}_{\mathrm{B}, \mathrm{ppm}}$ is the absorbed dose for boron-10 per ppm. Similarly, $\mathrm{D}_{\mathrm{N}}, \mathrm{D}_{\mathrm{H}}$, and $\mathrm{D}_{\gamma}$ indicate the absorbed doses for nitrogen, hydrogen, and gamma-ray, respectively. $C_{B}$ which is required to calculate the boron dose in each organ is the boron concentration (ppm). $\mathrm{CBE}_{\mathrm{B}}$ is the compound relative biological effectiveness for boron-10. In the dose evaluation, hypothetical values have been set according to the general protocol in BNCT. Each parameter used in the evaluation is summarized in Table 2.

The University of Tsukuba plans to implement a clinical study using the iBNCT for malignant melanoma. Therefore, the equivalent doses for normal tissue, skin, and tumors have been estimated by applying the protocol for malignant melanoma. In the simulation for the BNCT irradiation, the maximum equivalent dose of the skin is set as $15 \mathrm{~Gy}-\mathrm{Eq}$ and the concentration of boron-10 in blood is $25 \mathrm{ppm}$. In the estimation condition, irradiation time and maximum tumor dose have been estimated.

Table 2. Parameters required to the equivalent dose.

\begin{tabular}{|l|c|c|c|}
\hline & Tumor & $\begin{array}{c}\text { Normal } \\
\text { Tissue }\end{array}$ & Skin \\
\hline B-10 Ratio with Blood & 3.5 & 1.0 & 1.2 \\
\hline B-10 concentration $(\mathrm{ppm})$ & 88 & 25 & 30 \\
\hline CBE for BPA & 3.8 & 1.35 & 2.5 \\
\hline RBE $_{N}$ (Thermal Neutron) & \multicolumn{3}{|c|}{3.0} \\
\hline RBE $_{H}$ (Fast Neutron) & \multicolumn{3}{|c|}{3.0} \\
\hline Gamma-ray & \multicolumn{3}{|c}{} \\
\hline
\end{tabular}

\section{Results and discussions}

\subsection{Measurement results of the thermal neutron flux and gamma-ray dose rate}

Thermal neutron flux in the phantom has been measured by detecting the activation of the irradiated gold wires. Fig. 4 shows the distribution of the thermal neutron flux on the beam axis in the phantom. The maximum value is approximately $7.8 \times 10^{8}\left(\mathrm{~cm}^{-2} \mathrm{~s}^{-1}\right)$ at $20 \mathrm{~mm}$ depth of the phantom. The error margin of the measurement for the radio-activity of gold wire is approximately $\pm 7 \%$.

Fig. 5 shows the distribution of the gamma-ray dose rate on the beam axis. The irradiation condition for the average current of the proton beam is the same as the thermal neutron flux measurement. The maximum gamma-ray dose rate is approximately $2.8(\mathrm{~Gy} / \mathrm{h})$ at depth of $25 \mathrm{~mm}$ from the surface of the phantom. The error margin of the measurement with TLD is approximately \pm $12 \%$.

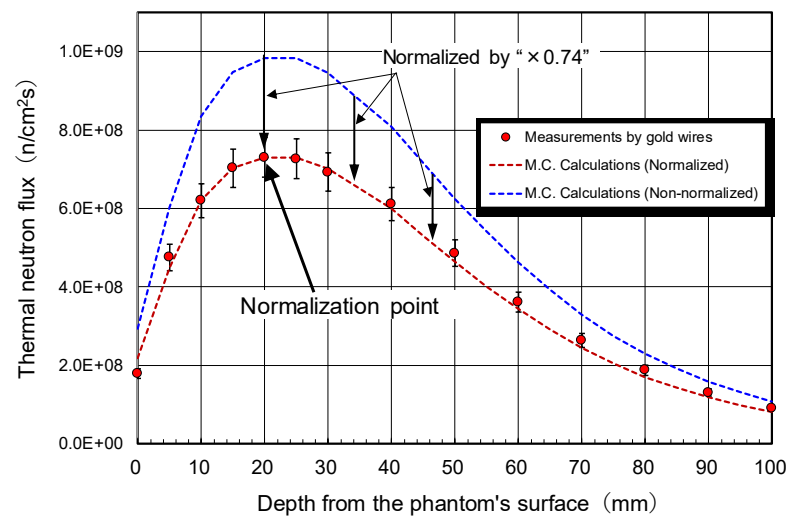

Fig. 4 Thermal neutron flux on the beam axis

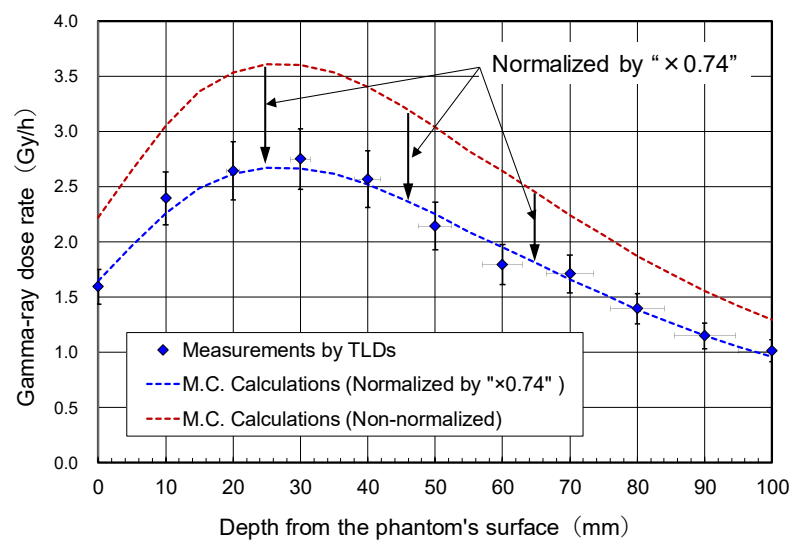

Fig. 5 Gamma-ray dose rate on the beam axis

\subsection{Dose estimations and irradiation time}

As described previously, the distributions for thermal neutron flux and gamma-ray dose rate in the phantom have been calculated by using PHITS. The value of the normalization factor was determined as 0.74 by dividing the experimental value of the maximum thermal flux $\left(7.80 \times 10^{8}\right)$ by the calculated value $\left(10.51 \times 10^{8}\right)$ at the same point (20-mm depth from the surface on the axis). The calculated values for the thermal neutron flux and gamma-ray dose rate have been normalized by the factor 
value. The calculated distributions for the thermal neutron flux and the gamma-ray dose rate are in good agreement with the measured distributions, as shown in Fig. 4 and Fig. 5. Based on these results, the distributions for four absorbed doses in the phantom have been determined by multiplying each calculated value for the absorbed doses with the normalization factor. Finally, the distributions for the equivalent doses for normal tissue, skin, and tumor regions have been estimated by applying the values of absorbed doses and each parameter listed in Table 2 to Equation 1.

From the estimation results, the irradiation time is approximately $44.10 \mathrm{~min}$, when $15.00 \mathrm{~Gy}-\mathrm{Eq}$ is administered to normal skin. According to this estimation condition, the maximum dose for the tumor and for normal tissue are approximately 54.64 Gy-Eq and 8.65 Gy-Eq, respectively. The estimation results prove that the iBNCT facility provides a neutron flux high enough for the treatment of malignant melanoma. This is because the irradiation time is less than $1 \mathrm{~h}$, and the prescribed dose administered to the tumor is more than $25.00 \mathrm{~Gy}$-Eq.

\section{Conclusions}

The development of the iBNCT, a linac-based neutron source for BNCT, is being carried out. The demonstration device of the iBNCT has been proven to succeed in generating high-intensity neutrons. Several experiments and measurements have been performed to evaluate the performance of the neutron source. The thermal neutron flux and the gamma-ray dose rate on the beam axis in a water phantom have been measured by phantom irradiation experiments. Furthermore, Monte Carlo simulations have been carried out to determine dose distribution administered to the irradiation field. The results of the experiments have demonstrated that the iBNCT can generate neutrons with sufficient intensity required for BNCT. To improve the stability and safety of the device further and to adopt the iBNCT device for actual BNCT clinical study, the device is being continuously advanced. Further experiments and analyses have been planned to understand the physical characteristics and biological effects of the neutron beam.

This work was supported by MEXT/JSPS KAKENHI (Grant number JP16H05236).

\section{References}

1. W. H. Sweet, J. Neuro-Oncol. 33, 19-26 (1997)

2. T. Aihara, N. Morita, N. Kamitani, H. Kumada, K. Ono, J. Hiratsuka and T. Harada, Int. J. Oncol. 19, 1437-1444 (2014)

3. A. J. Kreiner, M. Baldo, J.R. Bergueiro, D. Cartelli, W. Castell, V. Thatar Vento, et al., Appl. Radiat. Isot. 88, 185-189 (2014)

4. H. Kumada, F. Naito, K. Hasegawa, H. Kobayashi, T. Kurihara, K. Takada, T. Ohnishi, H. Sakurai, A. Matsumura and T. Sakae, Plasma and Fusion Research, 13 (2406006), 1-6 (2018)
5. H. Tanaka, Y. Sakurai, M. Suzuki, S. Masunaga, Characteristics comparison between a cyclotron-based neutron source and KUR-HWNIF for boron neutron capture therapy. Nucl. Instrum. Methods. Phys. Res. B 267, 1970-1977 (2009)

6. K. Yamamoto, H. Kumada, Y. Torii, T. Kishi, T. Yamamoto and A, Matsumura, Simple estimation method of gamma-ray dose using low neutronsensitive TLD (UD-170LS) for intra-operative boron neutron capture therapy (IOBNCT). Research and Development in Neutron Capture Therapy, (Monduzzi), 499-503 (2002)

7. H. Kumada and K. Takada, Therapeutic Radiology and Oncology, 2(50), 1-11 (2018) 